\title{
Closed-loop identification of MIMO systems: a new look at identifiability and experiment design
}

\author{
Alexandre Sanfelice Bazanella ${ }^{1,2}$, Michel Gevers ${ }^{1}$, Ljubiša Mišković ${ }^{1}$
}

\begin{abstract}
This paper addresses a question raised by a leading expert in the identification of multivariable systems: "Is it necessary to excite all reference signals for the identification of a multivariable system operating in closed loop with a linear time-invariant controller?" On the basis of earlier results on identifiability of closed-loop systems, he conjectured that this was necessary. We show that it is not, on the basis of a careful re-examination of the notions of identifiability and informative experiments for closed-loop systems.
\end{abstract}

\section{INTRODUCTION}

This paper re-examines the identifiability of closed-loop systems with a double objective: (i) clarify the differences between several definitions of identifiability and propose one that is both realistic and operational; (ii) establish conditions on the controller and on the external excitation that guarantee identifiability in the case of multi-input multi-output (MIMO) systems. In particular, our results shed new light on the experiment design question for MIMO systems.

Identifiability of linear systems operating in closed loop was a much studied problem in the 1970's and early 1980's. Results in [1], [2], [3], [4] seemed to fully answer all questions about the identifiability of closed-loop systems under different feedback configurations and for different assumptions about the excitation signals and the measured signals. Recently, there has been a renewed interest in closedloop identification in the context of least costly identification experiment for control [5], [6]. Briefly speaking, this concept refers to achieving a prescribed accuracy at the lowest possible price, measured in terms of the duration of the identification experiment, the perturbation induced by the excitation signal, or any combination of these.

In this context, recent work has focused on the specific contributions of the noise and of the external excitation signal to the accuracy of the parameters and the transfer function estimates, in both open and closed-loop identification [7], [8]. In this paper, we extend this work in the direction of MIMO systems by addressing a series of questions along the following lines: what are the exact experimental conditions that are required to make a MIMO system identifiable? is it possible to estimate a model even without external excitation? assuming that external excitation is required for

\footnotetext{
${ }^{1}$ Center for Systems Engineering and Applied Mechanics (CESAME), Université catholique de Louvain, B1348 Louvain la Neuve, Belgium \{gevers, miskovic, bazanella\}@csam. ucl.ac.be

${ }^{2}$ On leave from Dept of Electrical Engineering, Universidade Federal do Rio Grande do Sul, Brazil. Author partially supported by CAPES.

This paper presents research results of the Belgian Programme on Interuniversity Attraction Poles, initiated by the Belgian Federal Science Policy Office. The scientific responsibility rests with its author(s)
}

reasons of either identifiability or accuracy, is it necessary that all reference inputs be excited in a MIMO system?

In attempting to answer these and related questions, we have observed the need for a reassessment of the definitions of identifiability that were in use during the 1970's. Indeed, some of these definitions are unnecessarily demanding in terms of a realistic identification setup. For example, the main result in [2] states that in the case of a time-invariant controller a MIMO closed-loop system is strongly system identifiable if and only if all reference inputs are excited by persistently exciting signals. On the basis of such results, it is assumed by many in the system identification community that it is necessary to excite all external reference inputs if one wants to identify a system under closed-loop conditions. We show in this paper that this is not the case. Furthermore, our analysis will show that a certain accuracy level can be obtained using a variety of possible external excitation schemes; this includes, for example, excitation by just one of the external reference signals.

Our contribution, therefore, is essentially one of clarification. We recall the key definitions of identifiability and establish a clear distinction between identifiability of a model structure, and informativity (or richness) of the data set. The first notion depends only on the model structure chosen for the identification, and has nothing to do with the 'true system'. The conditions on the richness of the data, required for the existence of a unique global minimum of the identification criterion, are also a function of the chosen model structure. However, the data are collected on the true system; thus, whether these richness conditions are satisfied or not depends on the true system, the feedback configuration in a closed-loop setup, and the richness of the external data. For systems operating in closed loop, we show that if the controller is chosen of sufficient complexity with respect to the chosen model structure and if this model structure is globally identifiable, then a unique model can always be obtained without any external excitation, using information from the noise source only. Any desired accuracy of the parameter estimates can then be obtained by letting the data length $N$ be large enough. By adding external excitation at one or several of the reference inputs, one can achieve the desired accuracy in a shorter time.

The outline of our paper is as follows. In section II we present the key concepts of identifiability and of informativity of the data set. Section III establishes convergence conditions for the situation where the true system is contained in the model set. Section IV presents a numerical example. 


\section{IDENTIFIABILITY AND INFORMATIVITY}

\section{A. Definitions of identifiability}

The concept of identifiability involves the true system, the model structure used to estimate it, and the properties of the data set which, in a closed-loop setting, also depend on the feedback configuration. It has been given different contents in the econometrics and in the engineering literature [9]; the prevailing definition has also evolved over the years. [10] establishes a useful distinction between consistency-oriented and uniqueness-oriented definitions of identifiability.

Consistency-oriented identification deals with the question of whether the parameter estimate $\hat{\theta}_{N}$ converges to the 'true' parameter $\theta_{0}$ in some stochastic sense. This definition was prevalent in the 1970's when system identification was perceived as an exercise in finding the 'true system'. When identification began to be viewed as an exercise in finding the best approximate system within a model structure, convergence to a true $\theta_{0}$ became meaningless, since the model structure may very well not contain a description of the true system, i.e. there is no $\theta_{0}$. This is a severe limitation of the consistency-oriented definition of identifiability.

Uniqueness-oriented identification [11] deals with the question of whether the model structure is such that the identification criterion has a unique global minimum. This question has been addressed only for the asymptotic criterion, when the data length tends to infinity, since the criterion based on $N$ data is a random variable [10]. While this criterion no longer requires that the true system is in the model set, it is also not satisfactory because the existence of a unique minimum imposes conditions both on the model structure and on the data set used for identification. The conditions on the data set, in turn, depend on the true system and on the experimental conditions: open- or closed-loop configuration, richness of the signals.

From a users' point of view, it is important to work with a definition that clearly separates conditions on the model structure, which are entirely a user choice, and on the experimental conditions, which in many cases are also a user choice. In other words, an operational definition of identifiability is one in which the user can select a model structure with the knowledge that it is identifiable, and in which he/she can then select experimental conditions that are suitable for that model structure without any knowledge required about the true system. 'Identifiable model structure' means that it represents a given model for a unique value of the parameter $\theta$, while experimental conditions that are 'suitable for that model structure' means that with that model structure and these experimental conditions the identification criterion has, asymptotically, a unique global minimum. If in addition the true system can be exactly described within the chosen model structure, then the identified model will asymptotically converge to this true system.

In the sequel, we propose such a definition, in which we clearly separate between the concept of an identifiable model structure, and that of informative experiments with respect to that model structure.

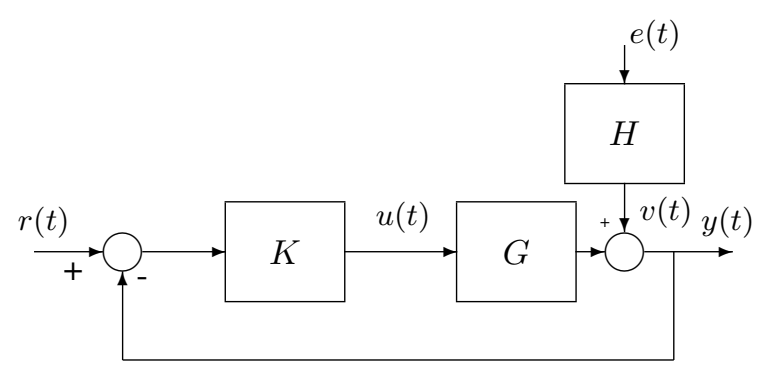

Fig. 1. Closed-loop configuration

\section{B. The identification setup}

We consider that the true system $\mathcal{S}$ is described by

$$
\mathcal{S}: y(t)=G\left(q^{-1}\right) u(t)+H\left(q^{-1}\right) e(t)
$$

where $G\left(q^{-1}\right)$ is a $p \times m$ stable, causal, rational transfer function matrix, and $H\left(q^{-1}\right)$ is a $p \times p$ stable and inversely stable transfer function matrix. The signal $y(t) \in \mathcal{R}^{p}$ is the output of the true plant, $u(t) \in \mathcal{R}^{m}$ is the control signal, while $e(t) \in \mathcal{R}^{p}$ is a zero-mean white noise input with covariance matrix $\Lambda$. This true system is under feedback control with a stabilizing controller $K$, a $m \times p$ causal rational transfer function matrix (see Figure 1):

$$
u(t)=K\left(q^{-1}\right)[r(t)-y(t)] .
$$

The system (1) is identified using a model structure parametrized by a vector $\theta \in \mathcal{R}^{d}$ :

$$
M(\theta): y(t)=G\left(q^{-1}, \theta\right) u(t)+H\left(q^{-1}, \theta\right) \varepsilon(t)
$$

The set of models $M(\theta)$, for all $\theta$ in some set $D_{\theta} \in \mathcal{R}^{d}$, defines the model set $\mathcal{M}: \mathcal{M} \triangleq\left\{M(\theta) \mid \theta \in D_{\theta}\right\}$. It is assumed that the loop transfer function $G\left(q^{-1}\right) K\left(q^{-1}\right)$ has a non-zero delay, both for $G\left(q^{-1}\right)$ and $G\left(q^{-1}, \theta\right), \theta \in D_{\theta}$. It is also assumed that $H(0)=I$. For brevity, we will most often drop the argument $q^{-1}$, thus referring to $G(\theta)$ and $H(\theta)$. The true system belongs to this model set, $\mathcal{S} \in \mathcal{M}$, if there is a $\theta_{0}$ such that $M\left(\theta_{0}\right)=\mathcal{S}$. In our definitions of identifiability, we shall not necessarily assume that $\mathcal{S} \in \mathcal{M}$. A model $[G(\theta) H(\theta)]$ uniquely defines the one-step-ahead predictor of $y(t)$ given all input/output data up to time $t$ :

$$
\begin{aligned}
\hat{y}(t \mid t-1, \theta) & \triangleq H^{-1}(\theta) G(\theta) u(t)+\left[I-H^{-1}(\theta)\right] y(t) \\
& =W_{u}(\theta) u(t)+W_{y}(\theta) y(t) \\
W_{u}(\theta) & =H^{-1}(\theta) G(\theta), W_{y}(\theta)=\left[I-H^{-1}(\theta)\right] .(5)
\end{aligned}
$$

For later use, we introduce the following notation:

$$
W(\theta) \triangleq\left[\begin{array}{ll}
W_{u}(\theta) & W_{y}(\theta)
\end{array}\right], \quad z(t) \triangleq\left[\begin{array}{l}
u(t) \\
y(t)
\end{array}\right]
$$

where $W$ denotes $p \times(m+p)$ rational transfer function matrix. The one-step-ahead prediction error is defined as:

$\varepsilon(t, \theta) \triangleq y(t)-\hat{y}(t \mid t-1, \theta)=H^{-1}(\theta)(y(t)-G(\theta) u(t))$.

Using a set of input-output data of length $N$ acquired in closed-loop operation, the estimate $\hat{\theta}_{N}$ is calculated via the 
prediction error criterion [12]:

$$
\hat{\theta}_{N}=\arg \min _{\theta \in D_{\theta}} \frac{1}{N} \sum_{t=1}^{N} \varepsilon^{T}(t, \theta) \varepsilon(t, \theta) .
$$

It defines the model $\hat{G}=G\left(\hat{\theta}_{N}\right), \hat{H}=H\left(\hat{\theta}_{N}\right)$. Under reasonable conditions [12], $\hat{\theta}_{N} \stackrel{N \rightarrow \infty}{\longrightarrow} \theta^{*} \triangleq \arg \min _{\theta \in D_{\theta}} \bar{V}(\theta)$, with

$$
\bar{V}(\theta) \triangleq E\left[\varepsilon^{T}(t, \theta) \varepsilon(t, \theta)\right] .
$$

If $\mathcal{S} \in \mathcal{M}$ and if $\hat{\theta}_{N} \stackrel{N \rightarrow \infty}{\longrightarrow} \theta_{0}$, the parameter error converges to a Gaussian random variable:

$$
\begin{aligned}
& \sqrt{N}\left(\hat{\theta}_{N}-\theta_{0}\right) \stackrel{N \rightarrow \infty}{\longrightarrow} N\left(0, P_{\theta}\right), \\
& P_{\theta}=\left.[I(\theta)]^{-1}\right|_{\theta=\theta_{0}}, \\
& I(\theta)=E\left(\psi(t, \theta) \Lambda^{-1} \psi(t, \theta)^{T}\right),
\end{aligned}
$$

with $\psi(t, \theta)=-\frac{\partial \varepsilon(t, \theta)}{\partial \theta}=\frac{\partial \hat{y}(t \mid t-1, \theta)}{\partial \theta}$. With $\varepsilon(t, \theta) \in \mathcal{R}^{p}$ and $\theta \in \mathcal{R}^{d}$, we define $\frac{\partial \varepsilon(t, \theta)}{\partial \theta}$ as a $d \times p$ matrix whose $(k, j)$-th element is $\frac{\partial \varepsilon_{j}(t, \theta)}{\partial \theta_{k}}$. The matrix $I\left(\theta_{0}\right)$ is called the information matrix.

\section{Identifiability of a model structure}

Here we adopt a uniqueness-oriented definition proposed in [12]; much of the paper serves to explain why this is an operational definition.

Definition 2.1: A parametric model structure $M(\theta)$ is globally identifiable at a value $\theta^{*}$ if $M(\theta)=M\left(\theta^{*}\right) \Rightarrow$ $\theta=\theta^{*}$. It is locally identifiable at $\theta^{*}$ if $M(\theta)=M\left(\theta^{*}\right) \Rightarrow$ $\theta=\theta^{*}$ for all $\theta$ in $\left|\theta-\theta^{*}\right|<\epsilon$ for some $\epsilon>0$. It is globally identifiable if it is globally identifiable at almost all $\theta^{*}$.

Comments: This definition is a property of the parametrization of $[G(\theta), H(\theta)]$. It tells us that if the model structure is globally identifiable at some $\theta^{*}$, then there is no other parameter value $\theta \neq \theta^{*}$ that yields the exact same model as $M\left(\theta^{*}\right)$. This definition does not say anything about possible convergence to this value $\theta^{*}$ or to a "true" parameter value $\theta_{0}$. The definition does not require that the system is in the model set, but it applies of course also to the situation where $\mathcal{S} \in \mathcal{M}$.

We now provide an equivalent definition of local identifiability. With $W$ defined as in (6), we introduce:

$$
\vec{W}(\theta) \triangleq\left[W_{1}(\theta) W_{2}(\theta) \ldots W_{p}(\theta)\right]
$$

where $W_{k}(\theta)$ denotes the $k$-th row of $W(\theta)$ with dimension $m+p$. Thus, $\vec{W}$ is a $p(m+p)$ row vector of transfer functions. The $d \times p$ matrix $\psi(t, \theta)$ can then be written as

$$
\psi(t, \theta)=\frac{\partial \vec{W}(\theta)}{\partial \theta} \operatorname{diag}[z(t), z(t), \ldots, z(t)]
$$

where $\frac{\partial \vec{W}(\theta)}{\partial \theta}$ is a $d \times p(m+p)$ matrix of transfer functions, while $\operatorname{diag}[z(t), z(t), \ldots, z(t)]$ is a $(p+m) p \times p$ blockdiagonal matrix with $z(t)$ in the diagonal blocks: see (6).

We now introduce the matrix $\Gamma_{1}(\theta) \in \mathcal{R}^{d \times d}$ :

$$
\Gamma_{1}(\theta) \triangleq \int_{-\pi}^{\pi} \frac{\partial \vec{W}\left(e^{j \omega}, \theta\right)}{\partial \theta} \frac{\partial \vec{W}^{H}\left(e^{j \omega}, \theta\right)}{\partial \theta} d \omega
$$

where $\vec{W}^{H}\left(e^{j \omega}\right)$ denotes $\vec{W}^{T}\left(e^{-j \omega}\right)$. The following result is an alternative definition for local identifiability of a model structure; see problem 4G.4 in [12] for a SISO version of this result.

Theorem 2.1: A parametric model structure $M(\theta)$ is locally identifiable at $\theta^{*}$ if $\Gamma_{1}(\theta)$ is nonsingular at $\theta^{*}$.

Proof: For $\theta$ close to $\theta^{*}$ we can write

$\vec{W}\left(e^{j \omega}, \theta\right)=\vec{W}\left(e^{j \omega}, \theta^{*}\right)+\left(\theta-\theta^{*}\right)^{T} \frac{\partial \vec{W}\left(e^{j \omega}, \theta\right)}{\partial \theta}+\sigma\left(\left|\theta-\theta^{*}\right|^{2}\right)$

where $\lim _{\theta \rightarrow \theta^{*}} \frac{\sigma\left(\left|\theta-\theta^{*}\right|^{2}\right)}{\left|\theta-\theta^{*}\right|}=0$. Assume that for $\theta$ close to $\theta^{*}$ we have $W\left(e^{j \omega}, \theta\right)=W\left(e^{j \omega}, \theta^{*}\right)$ for all $\omega$. It then follows from (16) that $\left(\theta-\theta^{*}\right)^{T} \frac{\partial \vec{W}\left(e^{j \omega}, \theta\right)}{\partial \theta}+\sigma\left(\left|\theta-\theta^{*}\right|^{2}\right)=0$ for all $\omega$. Multiplying this equation by its conjugate transpose and integrating over $\omega$ yields

$\left(\theta-\theta^{*}\right)^{T}\left\{\int_{-\pi}^{\pi} \frac{\partial \vec{W}\left(e^{j \omega}, \theta\right)}{\partial \theta} \frac{\partial \vec{W}^{H}\left(e^{j \omega}, \theta\right)}{\partial \theta} d \omega\right\}\left(\theta-\theta^{*}\right)=0$

If $\Gamma_{1}(\theta)$ is nonsingular, this implies $\theta=\theta^{*}$.

\section{Informative experiments for a model structure}

If a model structure is globally identifiable at some value $\theta^{*}$, it means that the model $M\left(\theta^{*}\right)$ cannot be represented by any other $M(\theta)$ within the model class. However, in order for an identification experiment to distinguish between $M\left(\theta^{*}\right)$ and any other model $M(\theta)$, it must be sufficiently informative with respect to that model structure.

We consider that the data are quasi-stationary [12]. An experiment shall then refer to the data generation mechanism which defines the asymptotic properties, and in particular the spectrum, of the joint input-output process $z(t)$ defined in (6). It does not refer to a particular set of $N$ data.

Definition 2.2: An experiment $z(t)$ is called informative with respect to a model set $\mathcal{M}$ if, for any two models $W^{(1)}$ and $W^{(2)}$ in that set,

$$
E\left\{\left|\left[W^{(1)}\left(q^{-1}\right)-W^{(2)}\left(q^{-1}\right)\right] z(t)\right|^{2}\right\}=0
$$

implies

$$
W^{(1)}\left(e^{j \omega}\right)=W^{(2)}\left(e^{j \omega}\right) \text { for almost all } \omega,
$$

where $W^{(i)}(q)$ are defined from a model by (5)-(6).

The definition means that there cannot be two different models within the model set that give exactly the same predictions, when fed with the same data. It is easy to show [12] that an experiment that yields $\Phi_{z}(\omega)>0$ for almost all $\omega$ is informative for all model structures.

Comments: (i) The definition of informative experiment is with respect to a given model set, not with respect to the true system, which may or may not belong to the model set. In an identification experiment, one typically first selects a globally identifiable model structure; this is a user's choice. Experimental conditions must then be selected that are informative with respect to that structure; this is again a user's choice. However, the data are generated by the true system, in open or in closed loop. Thus, the conditions that 
make a data set $z(t)$ informative with respect to some model structure depend on the true system and on the possible feedback configuration.

(ii) Informative experiments guarantee that no two different models within the set can generate the same predictions. A globally identifiable model structure additionally guarantees that almost all models in the set are represented by a unique parameter vector. The selection of a globally identifiable model structure, together with an experiment that is informative with respect to that model structure then guarantees that, asymptotically, the minimum of the criterion is almost surely unique, since it is unique at almost all $\theta^{*}$.

Consider now an identification experiment which generates data $z(t)$ with a power spectrum $\Phi_{z}(\omega)$. Combining (12) and (14) yields:

$$
\begin{aligned}
I(\theta) & =\frac{1}{2 \pi} \int_{-\pi}^{\pi} \frac{\partial \vec{W}\left(e^{j \omega}, \theta\right)}{\partial \theta} \operatorname{diag}\left[\Phi_{z}(\omega), \ldots, \Phi_{z}(\omega)\right] \\
\frac{\partial \vec{W}^{H}\left(e^{j \omega}, \theta\right)}{\partial \theta} d \omega & \\
= & \sum_{k=1}^{p} \frac{1}{2 \pi} \int_{-\pi}^{\pi} \frac{\partial W_{k}\left(e^{j \omega}, \theta\right)}{\partial \theta} \Phi_{z}(\omega) \frac{\partial W_{k}^{H}\left(e^{j \omega}, \theta\right)}{\partial \theta} d \omega
\end{aligned}
$$

We can now state the following theorem.

Theorem 2.2: Consider an identification experiment that generates data with spectrum $\Phi_{z}(\omega)$ and assume that a model structure $W(q, \theta)$ is used. Then $I\left(\theta^{*}\right)>0$ if the following two conditions hold:

(i) the model structure is locally identifiable at $\theta^{*}$;

(ii) $\Phi_{z}(\omega)>0$ for almost all $\omega$.

Proof: The result follows immediately by combining the positivity of $\Phi_{z}(\omega)$ and the nonsingularity of $\Gamma_{1}(\theta)$ that results from Theorem 2.1.

Comments: (i) The positivity of the information matrix at a value $\theta^{*}$ results from the combination of the two ingredients that are required to insure convergence to a unique minimum of an identification criterion at $\theta^{*}$ : the local identifiability of the model structure at $\theta^{*}$ and the informativity of the data with respect to that model structure.

(ii) The conditions of Theorem 2.2 are sufficient but they are by no means necessary. As we shall see later, condition (ii) is stronger than is needed. As for condition (i), we observe from the second expression in (20) that the nonsingularity of $I(\theta)$ can possibly be achieved with only one or a few of the $p$ terms in the sum.

\section{CONVERGEnCE TO THE TRUE SySTEM}

We now consider the situation where the system is in the model set: $\mathcal{S} \in \mathcal{M}$. Thus there exists a parameter vector $\theta_{0}$ such that $W\left(q^{-1}, \theta_{0}\right)=W^{0}\left(q^{-1}\right)$, where $W^{0}\left(q^{-1}\right)$ is the one-step ahead prediction filter corresponding to the true system: $W^{0} \triangleq\left[\begin{array}{ll}H^{-1} G & I-H^{-1}\end{array}\right]$. In addition, we assume that the model structure is globally identifiable at $\theta_{0}$. We combine these two assumptions in a single statement.

Assumption 1: The model structure $M(\theta)$ is such that $M\left(\theta_{0}\right)=\mathcal{S}$ for some $\theta_{0}$ and it is globally identifiable at $\theta_{0}$.
Comment: Assumption 1 does not necessarily imply that the model $M(\theta)$ has the same structure as the true system; it can be an overparametrization of $\mathcal{S}$. For example, consider that the true system is a single-input single-output ARX system described by $\left(1+0.8 q^{-1}\right) y(t)=0.5 u(t-1)+e(t)$. This system is contained in the model set $M(a, b, c)$ defined by the following ARMAX structure:

$$
M(a, b, c):\left(1+a q^{-1}\right) y(t)=b u(t-1)+\left(1+c q^{-1}\right) \varepsilon(t),
$$

which is globally identifiable at $\theta_{0}=(0.8,0.5,0)$.

Under Assumption 1 the Prediction Error identification algorithm converges to the unique value $\theta_{0}$ if the data are informative with respect to the chosen model structure.

Theorem 3.1: Consider a model structure that obeys Assumption 1 , and let $\bar{V}(\theta)$ be defined by (9). Then (i) $\theta_{0}$ is the unique minimum of $\bar{V}(\theta)$ if the data are informative with respect to the model structure $M(\theta)$.

(ii) $\theta_{0}$ is an isolated minimum of $\bar{V}(\theta)$ if $I\left(\theta_{0}\right)>0$. Proof: (i) Let $\hat{y}^{0}(t)$ denote the optimal predictor for the true system (1), such that for this true system: $y(t)=\hat{y}^{0}(t)+e(t)$. The asymptotic cost criterion $\bar{V}(\theta)$ can then be written as

$$
\begin{aligned}
\bar{V}(\theta) & =E\left[|y(t)-\hat{y}(t \mid t-1, \theta)|^{2}\right] \\
& =E\left[\left|\hat{y}^{0}(t)-\hat{y}(t \mid t-1, \theta)\right|^{2}\right]+\operatorname{tr} \Lambda .
\end{aligned}
$$

Clearly, $\theta_{0}$ is a global minimum of $\bar{V}(\theta)$, yielding $\hat{y}^{0}(t)=$ $\hat{y}\left(t \mid t-1, \theta_{0}\right)$ and $\bar{V}\left(\theta_{0}\right)=\operatorname{tr} \Lambda$. Let $\bar{\theta}$ be another global minimum. This implies that $E\left[\left|\hat{y}\left(t \mid t-1, \theta_{0}\right)-\hat{y}(t \mid t-1, \bar{\theta})\right|^{2}\right]=$ 0 . Since the data is informative with respect to $M(\theta)$, this implies $W(\bar{\theta})=W\left(\theta_{0}\right)$. By the global identifiability assumption at $\theta_{0}$, this in turn implies that $\bar{\theta}=\theta_{0}$.

(ii) We compute the first and second derivative of $\bar{V}(\theta)$.

$$
\bar{V}^{\prime}(\theta)=-2 E\left[\frac{\partial \hat{y}(t \mid t-1, \theta)}{\partial \theta} \varepsilon(t, \theta)\right]=0 \text { at } \theta=\theta_{0} .
$$

Here $\frac{\partial \hat{y}(t \mid t-1, \theta)}{\partial \theta}$ denotes a $d \times p$ matrix. As for $\bar{V}^{\prime \prime}(\theta)$, it is a $d \times d$ matrix whose $(k, j)$-th element is

$$
\begin{aligned}
{\left[\bar{V}^{\prime \prime}(\theta)\right]_{k, j}=} & -2 E\left\{\sum_{l=1}^{p} \frac{\partial^{2} \hat{y}_{l}(t \mid t-1, \theta)}{\partial \theta_{k} \partial \theta_{j}} \varepsilon_{l}(t, \theta)\right\}(23) \\
& +2 E\left\{\frac{\partial \hat{y}(t \mid t-1, \theta)}{\partial \theta_{k}} \frac{\partial \hat{y}^{T}(t \mid t-1, \theta)}{\partial \theta_{j}}\right\}
\end{aligned}
$$

Since $\varepsilon\left(t, \theta_{0}\right)=e(t)$ and since $e(t)$ is uncorrelated with the predictions up to time $t-1$, the first term of (23) is zero at $\theta=\theta_{0}$. The result then follows, since $\bar{V}^{\prime \prime}\left(\theta_{0}\right)=I\left(\theta_{0}\right)$.

We now present a range of experimental conditions that are informative with respect to the chosen model structure when the system operates under closed-loop control with a fixed (i.e. non-switching) controller.

\section{A. Identification without external excitation: $\mathbf{r}=\mathbf{0}$.}

We show that if one selects a model structure that obeys Assumption 1, and if the identification is performed in closed loop without external excitation (i.e. $r=0$ ) but with a controller $K\left(q^{-1}\right)$ of "sufficient complexity", then the asymptotic criterion $\bar{V}(\theta)$ has a unique global minimum 
at the value $\theta_{0}$ for which $M\left(\theta_{0}\right)=\mathcal{S}$. First we state two technical lemma's that will be needed subsequently.

Lemma 3.1: [13] Let $D(z)$ be a row reduced ${ }^{1}$ polynomial matrix of full row rank and let $q(z)=p(z) D(z)$ where $p(z)$ is any polynomial row vector. Then

$$
\operatorname{deg} q(z)=\max _{j: p_{j}(z) \neq 0}\left[\operatorname{deg} p_{j}(z)+k_{j}\right]
$$

where the degree of a vector is defined as the highest degree of any of its elements, $p_{j}(z)$ is the $j$-th entry of $p(z)$, and $k_{j}$ is the degree of the $j$-th row of $D(z)$.

Lemma 3.2: [15] Consider the Diophantine equation

$$
A X+B Y=0
$$

where $A, B, X, Y$ are polynomial matrices in the variable $z$, with $X$ and $Y$ given, and $A$ and $B$ unknown. The general solution of this equation can be written as

$$
B=-P X_{1}, A=P Y_{1},
$$

where $X_{1}, Y_{1}$ are a coprime solution of $Y_{1} X=X_{1} Y$ and $P$ is an arbitrary polynomial matrix.

Now, consider Definition 2.2 and assume that

$$
E\left\{\left|\left[W\left(\theta_{1}\right)-W\left(\theta_{2}\right)\right] z(t)\right|^{2}\right\}=0 .
$$

Remembering (6), introducing $\Delta W_{u}\left(\theta_{1}, \theta_{2}\right) \triangleq W_{u}\left(\theta_{1}\right)-$ $W_{u}\left(\theta_{2}\right)$ and $\Delta W_{y}\left(\theta_{1}, \theta_{2}\right) \triangleq W_{y}\left(\theta_{1}\right)-W_{y}\left(\theta_{2}\right)$, and substituting $u(t)=-K y(t)$, we can rewrite (27) as

$$
E\left\{\left|\left[\Delta W_{y}\left(\theta_{1}, \theta_{2}\right)-\Delta W_{u}\left(\theta_{1}, \theta_{2}\right) K\right] y(t)\right|^{2}\right\}=0 .
$$

Since $\Phi_{y}(\omega)$ is generically nonsingular for almost all $\omega$, this implies, using Parseval's theorem and considering $\Delta W_{y}$, $\Delta W_{u}$ and $K$ as rational transfer function matrices in the variable $z$, that

$$
\Delta W_{y}-\Delta W_{u} K=0
$$

Now let

$$
\left[\begin{array}{ll}
\Delta W_{y} & -\Delta W_{u}
\end{array}\right]=D^{-1}\left[\begin{array}{ll}
B & A
\end{array}\right]
$$

be a left coprime polynomial factorization with $D(z)$ row reduced and let $k_{\max }$ be the largest row degree of the polynomial matrix $\left[\begin{array}{ll}B & A\end{array}\right]$. We can then state the main result of this subsection.

Theorem 3.2: Let the true system (1) be identified in closed loop with a feedback controller $u(t)=-K\left(q^{-1}\right) y(t)$ without external excitation, using a model structure that obeys Assumption 1. Let $c_{\text {min }}$ denote the minimum observability index of any irreducible representation of $K\left(q^{-1}\right)$. Then $\theta_{0}$ is the unique global minimum of the asymptotic criterion $\bar{V}(\theta)$ if $c_{\min }>k_{\max }$.

Proof: Let $K=X Y^{-1}$ be a right coprime factorization. Then, using equation (30), (29) can be written as:

$$
A X+B Y=0
$$

\footnotetext{
${ }^{1}$ See [14] for properties of polynomial matrices, such as coprime factorizations, column- or row-reduced matrices, etc.
}

where $A \in \mathbb{R}^{p \times m}[z], B \in \mathbb{R}^{p \times p}[z], X \in \mathbb{R}^{m \times p}[z], Y \in$ $\mathbb{R}^{p \times p}[z]$. By Lemma 3.2, the general solution of (31) is

$$
\left[\begin{array}{ll}
B & A
\end{array}\right]=P\left[\begin{array}{ll}
-X_{1} & Y_{1}
\end{array}\right],
$$

where $K=Y_{1}^{-1} X_{1}$ is a left coprime row reduced factorization of $K, P$ is an arbitrary polynomial matrix in $\mathbb{R}^{p \times m}[z]$, while $c_{\text {min }}$ is the smallest row degree of the matrix $\left[\begin{array}{ll}-X_{1} & Y_{1}\end{array}\right]$ [14]. Now, if $c_{\min }>k_{\max }$ then by Lemma 3.1 the only solution to (31) is obtained by setting $P=0$ in (32), which implies $\Delta W_{y}=0$ and $\Delta W_{u}=0$ by (30). The result then follows from part (i) of Theorem 3.1.

Comments: (i) The choice of a controller that satisfies the complexity requirement of the Theorem depends only on the model structure chosen by the user; (ii) A similar result can be found in [3].

\section{B. Identification with external excitation: $\mathbf{r} \neq \mathbf{0}$}

We have just shown that closed-loop identification can always be performed without external excitation, provided a controller of sufficient complexity is chosen. However, by doing so one may have to collect many data to arrive at a prescribed level of accuracy. Applying external excitation signals adds contributions to the information matrix $I(\theta)$, and therefore decreases the covariance matrix $P_{\theta}$ for a same number of data $N$.

Condition (27) can be rewritten as follows, using Parseval's theorem:

$$
\begin{aligned}
& \operatorname{tr}\left\{\int_{-\pi}^{\pi}\left[\Delta W_{u}\left(e^{j \omega}, \theta_{1}, \theta_{2}\right) \quad \Delta W_{y}\left(e^{j \omega}, \theta_{1}, \theta_{2}\right)\right] \Phi_{z}(\omega)\right. \\
& \left.\times\left[\Delta W_{u}\left(e^{j \omega}, \theta_{1}, \theta_{2}\right) \quad \Delta W_{y}\left(e^{j \omega} \theta_{1}, \theta_{2}\right)\right]^{H}\right\}=0 .
\end{aligned}
$$

This implies $\Delta W_{u}\left(e^{j \omega}, \theta_{1}, \theta_{2}\right) \equiv 0$ and $\Delta W_{y}\left(e^{j \omega}, \theta_{1}, \theta_{2}\right) \equiv$ 0 if $\Phi_{z}(\omega)>0$ for almost all $\omega$. The conditions under which this holds depend on the experimental conditions, and therefore on the true system $\left[\begin{array}{ll}G & H\end{array}\right]$ and on the controller $K$. The expression of $z$ as a function of the true closed-loop system is as follows:

$$
z \triangleq\left(\begin{array}{l}
u \\
y
\end{array}\right)=\left[\begin{array}{cc}
I & K \\
-G & I
\end{array}\right]^{-1}\left[\begin{array}{cc}
K & 0 \\
0 & H
\end{array}\right]\left(\begin{array}{l}
r \\
e
\end{array}\right)
$$

Since $G K$ is strictly causal, the first matrix is always nonsingular. Assuming that $K$ has full normal rank, then $\Phi_{z}(\omega)>0$ for almost all $\omega$ if $\Phi_{r}(\omega)>0$ for all $\omega$. However, while this is a sufficient condition for the generation of informative data, it is by no means necessary. A complete analysis of the conditions that make closed-loop data, obtained with a time-invariant controller, informative with respect to a MIMO model structure is based on the analysis of the linear dependencies of the matrix (see (33)):

$$
\left[\Delta W_{u}\left(e^{j \omega}, \theta_{1}, \theta_{2}\right) \quad \Delta W_{y}\left(e^{j \omega}, \theta_{1}, \theta_{2}\right)\right] \Phi_{z}(\omega)
$$

when the data are generated by (34). This type of analysis for single-input single-output systems is performed in [16]. Informative data can be obtained from noise excitation only (if $K$ is sufficiently complex), from some or all of the reference signals, or from a combination of reference signals 
and noise excitation. A full-scale analysis is beyond the scope of this conference paper.

In [2] it was shown that, in the case of a time-invariant controller, strong system identifiability can be achieved only if all references are excited. However, strong system identifiability means that the model estimate must converge to the true system for all model structures that can represent the true system. This requirement is too strong to be practical, since in practice the user selects a particular model structure.

In the next section, we illustrate with a 2-input 2-output example that it is possible to achieve the prescribed level of accuracy by exciting one of the reference signals only.

\section{Simulation Results}

The following ARX structure is considered:

$$
\begin{aligned}
& A_{1} y_{1}(t)=B_{11} u_{1}(t)+B_{12} u_{2}(t)+e_{1}(t) \\
& A_{2} y_{2}(t)=B_{21} u_{1}(t)+B_{22} u_{2}(t)+e_{2}(t)
\end{aligned}
$$

with $A_{1}=1-0.9535 q^{-1}, B_{11}=0.744 q^{-1}, B_{12}=$ $-0.8789 q^{-1}, A_{2}=1-0.9329 q^{-1}, B_{21}=0.5786 q^{-1}$, $B_{22}=-1.302 q^{-1} ; e_{1}$ and $e_{2}$ are mutually independent zeromean white Gaussian noises with $\lambda_{1}=\lambda_{2}=0.05$. We consider the parameter vector $\theta=\left(b_{11}, b_{12}, b_{21}, b_{22}, a_{1}, a_{2}\right)^{T}$. The plant is controlled by a $2 \times 2$ PI controller which stabilizes the plant without other performance consideration:

$$
K\left(q^{-1}\right)=\left(\begin{array}{cc}
\frac{0.55-0.45 q^{-1}}{\left(1-q^{-1}\right)} & \frac{0.15-0.08 q^{-1}}{\left(1-q^{-1}\right)} \\
\frac{0.24-0.18 q^{-1}}{\left(1-q^{-1}\right)} & \frac{-0.35+0.33 q^{-1}}{\left(1-q^{-1}\right)}
\end{array}\right) .
$$

We want to attain the desired accuracy by exciting one of the reference signals only. Parametrize the reference signal spectra as follows [5],

$$
\Phi_{r_{i}}(\omega)=R_{r_{i}}(0)+2 \sum_{k=1}^{m} R_{r_{i}}(k) \cos (k \omega) \geq 0 \quad \forall \omega
$$

where $i=1,2$, and $m$ is a positive integer, and consider the following experiment design problem [6]:

$$
\begin{aligned}
\underset{\Phi_{r_{1}}, \Phi_{r_{2}}}{\operatorname{minimize}} & E_{r_{2}} \\
\text { subject to } & \operatorname{tr} P_{\theta} \leq \gamma \\
& \frac{1}{2 \pi} \int_{-\pi}^{\pi} \Phi_{u_{i}}(\omega) d \omega \leq E_{u_{i}}, \quad i=1,2 \\
& \frac{1}{2 \pi} \int_{-\pi}^{\pi} \Phi_{r_{2}}(\omega) d \omega \leq E_{r_{2}} \\
& \Phi_{r_{1}}(\omega)=0, \Phi_{r_{2}}(\omega) \geq 0 \quad \forall \omega
\end{aligned}
$$

where $\gamma=1, E_{u_{1}}=E_{u_{2}}=0.4$ and $m=50$ in (37). The solution $r_{2}(t)$ with the minimal energy required to satisfy the constraints yields $E_{r_{2}}=1.777$. A Monte-Carlo simulation is performed to verify the results of the experiment design. $r_{1}(t)$ is kept equal to zero, while $r_{2}(t)$ is generated according to the solution of the optimization problem (38). The data length is $N=1000$. The trace of $P_{\theta}$ computed by 500 Monte-Carlo runs is $\operatorname{tr} P_{\theta}=1.047$. This slightly exceeds the bound $\gamma=1$; this is due to the fact that the optimization problem is solved using the asymptotic covariance expression (see (11)-(12)), while the Monte-Carlo computations are based on estimates obtained from 1000 data. The simulation confirms that it is not necessary to excite both reference signals to attain a given accuracy level.

\section{CONCLUSIONS}

The origin of this work was to take a critical look at a statement made to us: "For the identification of a MIMO system based on closed-loop data collected with a timeinvariant controller, it is necessary to excite all reference inputs." We have shown that this is not the case. In analyzing this question, we have made a clear distinction between identifiability of a model structure, and selection of experimental conditions that make the data informative with respect to that structure. We have shown that, once a model structure is selected, the user can always chose a controller of sufficient complexity that will make the data informative with respect to that model structure. We have also shown that the addition of reference signal excitation may serve to improve the quality of the parameter estimates, but that several excitation scenarios can typically be considered.

\section{REFERENCES}

[1] T. Söderström, I. Gustavsson, and L. Ljung, "Identifiability conditions for linear systems operating in closed loop," International Journal of Control, vol. 21, pp. 243-255, 1975.

[2] T. Söderström, L. Ljung, and I. Gustavsson, "Identifiability conditions for linear multivariable systems operating under feedback," IEEE Trans. Automatic Control, vol. AC-21, pp. 837-840, 1976.

[3] T. S. Ng, G. C. Goodwin, and B. D. O. Anderson, "Identifiability of MIMO linear dynamic systems operating in closed loop," Automatica, vol. 13, pp. 477-485, 1977.

[4] B. D. O. Anderson and M. Gevers, "Identifiability of linear stochastic systems operating under linear feedback," Automatica, 18, vol. 2, pp. 195-213, 1982.

[5] X. Bombois, G. Scorletti, M. Gevers, P. Van den Hof, and R. Hildebrand, "Least costly identification experiment for control," Automatica, vol. 42, no. 10, pp. 1651-1662, October 2006.

[6] H. Jansson and H. Hjalmarsson, "Optimal experiment design in closed loop," in 16th IFAC World Congress on Automatic Control, paper 04528, July 2005.

[7] X. Bombois, M. Gevers, and G. Scorletti, "Open-loop versus closedloop identification of Box-Jenkins models: a new variance analysis," in CD-ROM Proc. of the joint CDC-ECC Conference, Seville, Spain, 2005, pp. 3117-3122.

[8] L. Mišković, A. Karimi, D. Bonvin, and M. Gevers, "Direct closedloop identification of 2 × 2 systems: variance analysis," in $C D$ ROM Proc. of 14-th IFAC Symp. on System Identification, Newcastle, Australia, 2006, pp. 873-878.

[9] V. V. Nguyen and E. F. Wood, "Review and unification of linear identifiability concepts," SIAM Review, vol. 24, no. 1, pp. 34-51, January 1982

[10] L. Ljung, "On consistency and identifiability," Mathematical Programming Study, vol. 5, pp. 169-190, 1976.

[11] R. Bellman and K. J. Åström, "On structural identifiability," Mathematical Biosciences, vol. 7, pp. 329-339, 1970.

[12] L. Ljung, System Identification: Theory for the User, 2nd Edition. Englewood Cliffs, NJ: Prentice-Hall, 1999.

[13] G. D. Forney, "Minimal bases of rational vector spaces, with applications to multivariable linear systems," SIAM Journal of Control, vol. 13, pp. 493-520, 1975.

[14] T. Kailath, Linear Systems. Englewood Cliffs, New Jersey: PrenticeHall, 1980

[15] V. Kučera, Discrete linear control: the polynomial approach. John Wiley, 1979.

[16] M. Gevers, A. Bazanella, and L. Mišković, "Identifiability and informative experiments in open and closed-loop identification," in 'Modeling, Estimation and Control: Festschrift in honor of Giorgio Picci on the occasion of his sixty-fifth Birthday' A. Chiuso, A. Ferrante and S. Pinzoni Eds., ser. Lecture Notes in Control and Information Sciences. Springer Verlag, 2007 